\title{
DEPARTAMENTOS DE ORIENTACIÓN: UN ANÁLISIS DEL ROL ORIENTADOR Y DE LAS NUEVAS FIGURAS ASESORAS (1)
}

\author{
GUIDANCE FEPARTMENTS: AN STUDY ABOUT CONSULTING \\ WORK AND ADVISER
}

\author{
Félix Loizaga Latorre* \\ Universidad de Deusto
}

\begin{abstract}
RESUMEN
Este artículo trata de la situación actual de los Departamentos de Orientación (D.O.), subrayando la figura asesora del orientador. Consta de tres apartados: el primero analiza el origen de los D.O. en los años ochenta, y su puesta en práctica durante la década de los noventa; siguiendo las directrices de la LOGSE. La globalización actual, como telón de fondo, implica una reorientación de los D.O. Las figuras asesoras se encuentran desbordadas, en relación a la diversidad de tareas y a la población a la que orientan. En el segundo apartado se presentan las líneas futuras de trabajo en las que deberá incidirse para mejorar la calidad y la atención en los centros. Finalmente, en el tercero se plantea incorporar nuevas figuras asesoras con diferentes funciones para optimizar la eficacia de los D.O.
\end{abstract}

Palabras claves: Departamento de orientación, educación secundaria.

\begin{abstract}
This article reflects on the current situation of the Guidance Departments, putting emphasis on the figure of the adviser. It consists of three sections. The first one analyses the origin of the G.D. in the 80 's, and the their implementation during the decade of the 90's; with reference to the LOGSE. The present globalization, as a backcloth, leads to the Guidance Departments having to be reoriented so as to be efficient in their management. The advisers are "overwhelmed with their duties", with regard to the variety of tasks and the population they give advise to. On the second section, the future lines of work on which it will be necessary to touch upon, in order to improve the quality and the attention in
\end{abstract}

* Profesor de la Universidad de Deusto (Departamento de Psicopedagogía, y Psicología). mparte: Practicum de Psicología de la Educación, Psicología Clínica del Desarrollo, y Psicología de la Personalidad. Profesor-Tutor UNED Bizkaia. Email: floizaga@fice.deusto.es. Ha sido Técnico en Orientación del País Vasco durante los años 1988-1993, y profesor-orientador en ESO durante los años 1994-1999. Líneas de Investigación: Adolescencia, sucesos vitales y psicopatología; Educación sexual y Adolescencia; Familia y Adopción. 
the institutions, are introduced. And on the third one, it is proposed to include new figures of guidance into the G. D. with different tasks to improve the efficacy of the G. D.

Key words: Guidance Department, secondary education.

\section{Del Libro Blanco a la realidad del año dos mil. La visión retrospectiva}

\section{Introducción}

Cuando el Ministerio de Educación planteó el Libro Blanco (MEC, 1989), dio indicaciones sobre cómo serían los Departamentos de Orientación (D.O.). Estos aparecen como uno de los niveles de intervención en los centros junto con la tutoría y los equipos de apoyo externos. Los D.O. dependerán así de los centros educativos y tendrán como eje la figura del profesor orientador como experto, siendo un recurso "intracentro" (MEC, 1990).

A principios de los años 90 este planteamiento generaba gran ilusión y expectativa, ya que se esperaba que podrían ayudar a las necesidades de los centros al establecer una persona responsable para que los contenidos de orientación y tutoría se plasmaran concretamente.

El perfil del orientador respondía a las necesidades e intereses de aquellos años, en especial a tareas como la tutoría, la orientación escolar y personal del alumnado y el apoyo a las necesidades educativas especiales (MEC, 1992). En principio, casi todo encajaba en esas necesidades y en las circunstancias psicosociales y psicoeducativas de la sociedad de esos años. Otras figuras se perfilaban ya entonces como posibles componentes de los departamentos, especialmente el profesor de apoyo a la educación especial, también llamado profesor especialista en pedagogía terapéutica.

Los tutores y tutoras ya venían trabajando, como agentes de la orientación. Atendían a su alumnado de manera individual y grupal y también a sus familias. Y esto desde hacía tiempo, pues los Institutos de Ciencias de la Educación (ICE), e encargaban de asesorarles, junto con otros colectivos pedagógicos, asociaciones y equipos externos, cuando los centros no tenían aún organizada la estructura del Departamento de Orientación.

Se hablaba de Departamentos de Orientación desde hacía muchos años. Uno de los primeros libros que respondían a ese título apareció en 1970 (Iturbe y Del Carmen, 1973), pero los Departamentos se implantaron y regularon legalmente a partir de 1995 y posteriormente irían ajustándose otros perfiles y figuras hasta llegar a la configuración actual.

En este trabajo se pretende reflexionar sobre las consecuencias de haber elaborado un planteamiento teórico de configuración de los D.O. con la información y la documentación de los años ochenta, para ser implementado diez años después. En esta década han ocurrido demasiados cambios estructurales como para no tenerlos en cuenta:

1. La evolución de las corrientes sociales: emigración, consumo (también el consumo "escolar"), nuevos derechos sociales, afianzamiento de la sociedad democrática, competencias educativas transferidas a las Autonomías, etc.

2. Las nuevas tecnologías de la información y comunicación (internet, redes...) y su repercusión en las técnicas de enseñanza y orientación. 
3. El planteamiento de la estructura y los valores de la familia: diferentes tipos de familias, y por tanto diferentes tipos de hijos e hijas que acuden a los centros escolares.

Por otra parte, se reflexionará sobre las necesidades psicosociales y psicoeducativas a las que pretenden responder los Departamentos de Orientación en los próximos años, y por tanto los recursos humanos necesarios para las tareas orientadoras. También se indican las funciones que deberán realizar esas personas para que lleguen de verdad a orientar. Algo muy diferente a lo que ocurre actualmente en España, donde los orientadores y las orientadoras, con gran esfuerzo por su parte, intentan, sin conseguirlo, abarcarlo todo, generándose la consiguiente frustración y soledad.

\section{Lo que no se preveía en la década de los ochenta cuando se planteó la idea de los Departamentos de Orientación}

Fundamentalmente, las consecuencias de un mundo más "globalizado":

1. Un aumento en la diversidad del alumnado en los centros educativos (emigrantes, minorías étnicas, alumnos y alumnas con diferentes organizaciones familiares...).

2. Diferentes ideas de libertad, convivencia, orden..., en definitiva, de la idea de educar dentro y fuera de las aulas.

3. Nuevas metodologías de enseñanza y orientación

4. Un aumento de los itinerarios educativos, así como del número de carreras y estudios existentes.

Todo ello ha configurado un marco distinto del esperado en 1990 para los D.O.:

1. El concepto de necesidad educativa especial (n.e.e.), requiere un nuevo planteamiento. La diversidad es tan amplia que el concepto de n.e.e. puede llegar a vaciarse, ya que en principio abarca a todos, y puede acabar no respondiendo a nadie.

2. La enseñanza-aprendizaje requiere mucho más que metodologías y didácticas; por ejemplo saber aconsejar y orientar a las distintas clases, alumnos y familias.

3. La orientación no puede pasar por alto las nuevas tecnologías. Las personas piden hoy una orientación personalizada pero exigen a la vez la posibilidad de una orientación a distancia.

4. Ante el cúmulo de información, el alumnado y las familias piden cada vez más orientación sobre los tipos, lugares y características de los posibles estudios. Hoy no delimitados al ámbito local o autonómico.

\section{Sobre los Departamentos de Orientación en España y en otros países europeos: más diferencias que semejanzas}

La misión fundamental de los D.O. es "participar, animar, asesorar y trabajar conjuntamente en los distintos foros de decisión de la intervención educativa en los centros (...) y colaborar con sus aportaciones en la construcción de proyectos, diseños de evaluación, criterios de intervención y, en definitiva, en la tarea educativo-orientadora del alumnado" (Motoso, 2002, p. 83). 
Si algo queda claro, es que los D.O. en Europa, como los sistemas educativos en general, están configurados de manera muy diferente. Algunos tienen estructuras muy descentralizadas (dependientes de ayuntamientos o instituciones locales), mientras que otros tienen organizaciones dependientes de sus gobiernos nacionales (Álvarez, 1999; 2002).

Algunas consideraciones importantes que nos debieran hacer pensar sobre nuestro modelo son: las siguientes:

1. En nuestro país, las cuatro áreas de intervención básicas son:

a) la orientación en los procesos de enseñanza-aprendizaje;

b) orientación profesional;

c) atención a la diversidad, y

d) orientación para la prevención y el desarrollo (Bisquerra, 2002).

Y estas áreas, que en España recaen directamente bajo la responsabiliad del orientador, en otros países se reparten entre distintos profesionales.

2. Los perfiles profesionales y la formación de base difiere también sustancialmente. En nuestro país, los profesionales que coordinan los departamentos son licenciados en Psicología, Pedagogía o Psicopedagogía: distintas formaciones para la realización de una única, aunque compleja, actividad laboral. En otros países, ocurre que o bien no existe como tal alguna de estas tres licenciaturas (especialmente Psicopedagogía), o bien se exige una especialización tras la licenciatura, o bien finalmente puede optarse a esta actividad desde formaciones tan distintas como la sociología o incluso la ingeniería.

De ello se deduce, por tanto, que hay diferentes modelos, así como diferentes estructuras administrativas, e igualmente diferentes capacitaciones profesionales, funciones y poblaciones que atender. El concepto de D.O. en España, aunque difiere entre las distintas Comunidades Autónomas, es único con relación al europeo.

\section{De la disonancia entre las funciones que se están realizando y lo que se preveía en los años 80: la complejidad de los colectivos a los que se atienden y perfiles de los componentes de los D.O.}

Ya se ha comentado previamente que los departamentos tienen cuatro áreas de intervención básicas, con muy diferentes colectivos. Algunos de estos colectivos atendidos por el D.O. y en especial por los orientadores, son los siguientes:

a) profesorado (y, de manera específica, tutores);

b) alumnado (con diferentes problemáticas: intelectual, física, conductual, emocional, desarrollo...; con difiultades de motivación escolar; los que llamamos "normales", pero que a su vez también presentan dificultades);

c) familias de diversos tipos;

d) equipos internos (directivos, departamentos y seminarios, el propio departamento orientación...);

e) equipos externos (unidades de trabajo social, minorías, centros de apoyo al empleo...). 
Lo que no podía adivinarse era la complicación de tareas, funciones y nuevas necesidades que iban a ir surgiendo, derivadas de la interacción entre las funciones y colectivos. Aparecen así grupos de "diversificación curricular", "aulas de tareas", "aulas para alumnos con baja motivación", y otras necesidades derivadas de la nueva formación profesional, como las asignaturas de formación y orientación laboral. Se trata de grupos y personas que demandan atención de los D.O.

Para poder cubrir estas necesidades, las administraciones educativas han propuesto nuevas figuras, algunas de ellas de perfil profesional poco definido, y cubiertas por profesionales sin formación específica. La administración educativa ha propuesto cubrir las plazas de los D.O. con el exceso de otras plazas docentes debido al descenso del número de alumnos y alumnas en los centros

Ahora bien, las tareas de los D.O. se asientan sobre funciones propias de asesoramiento, orientación y desarrollo de programas; es decir, actividades específicas de profesionales con formación superior. Y además, ocurre que muchos centros tienen un solo orientador o una sola orientadora a pesar del elevado número de alumnos, profesores y familias que tiene que atender.

Dos pilares conforman las actuaciones de los D.O.: el asesoramiento-orientación y la docencia a alumnado con y sin necesidades educativas especiales. El primero depende de manera directa del orientador. El otro, del resto de los profesionales del D.O. que se dedican fundamentalmente a la docencia de alumnos con n.e.e., alumnos con problemas de motivación. y otros grupos específicos. Es decir, profesorado de pedagogía terapéutica, de diversificación, de tareas y de asignaturas de F.O.L (Formación y Orientación Laboral). En algunas Comunidades Autónomas los trabajadores sociales hacen funciones de apoyo socioeducativo a alumnos de determinados grupos sociales.

En consecuencia, muchas de las funciones asignadas por el MEC no han podido ser suficientemente desarrolladas, puesto que los orientadores deben coordinar, asesorar, evalúar, formar y orientar: demasiadas funciones para un solo profesional.

\section{Del apoyo de los Equipos Interdisciplinarios de Sector y otros organismos a los D.O.}

En muchas Comunidades Autónomas, los equipos externos de apoyo a los centros escolares fueron creados previamente a los D.O., ya en 1977 (Servicio de Orientación Escolar y Vocacional, SOEV), y posteriormente, los equipos de apoyo actuales (Boza, 2001), siempre muy cercanos a las estructuras políticas, centrales y autonómicas). Los actuales equipos interdisciplinares externos aparecen como estructuras de coordinación y apoyo a los centros (en especial, en temas como las n.e.e., las nuevas tecnologías de la información y comunicación, y didácticas específicas). Algunas Comunidades incluían técnicos para sus lenguas cooficiales. Y, excepcionalmente, técnicos específicos para orientación (País Vasco y otras). El sistema externo nació para apoyar y formar a los centros, así como para evaluar a alumnos con n.e.e.), pero la actuación más directa sobre alumnos, tutores y familias se reserva normalmente a los D.O. internos

Los centros externos se mantienen como vías de información, formación y transmisión de la comunicación de las diferentes administraciones educativas. También realizan la eva- 
luación de necesidades y proponen recursos específicos para ayudar a los centros y a su alumnado. Pero también es verdad que las ayudas llegan después de amplios trabajos que los centros desarrollan, trabajos apoyados y realizados frecuentemente por los D.O., que asumen estas tareas como un elemento más en su que hacer a lo largo de los cursos escolares (Boza, 2001).

En realidad, las estructuras externas ayudan al sistema, pero generan trabajo al demandar sistemáticamente informes escritos previos a cualquier ayuda económica $\mathrm{y}$, en ocasiones, también, pedagógica. Otras veces, estos equipos externos, proponen la realización de nuevas tareas que deben ser asumidas por los centros y sus D.O.

Por otra parte, los sistemas locales se van haciendo cada vez más complejos. Muchos ayuntamientos tienen departamentos de educación específicos que demandan actuaciones sobre los centros, y frecuentemente las ONG reclaman programas sobre los mismos (campañas de solidaridad, salud, etc.), todos ellos a través de los D.O. En definitiva, lo que en principio iba a ser un apoyo y una ayuda, se ha convertido ahora en nuevas obligaciones: organizar, diseñar, planificar...

\section{Del exceso de funciones y tareas sobre una misma figura}

"No tengo tiempo" o "no puedo con todo" son las frases más repetidas. En realidad, lo que ocurre es que hay un exceso de funciones y tareas: la orientación, la evaluación, el asesoramiento y el diseño de programas están directamente ligados a la persona del orientador. $\mathrm{Y}$ en no pocas ocasiones se incluye la docencia (por ejemplo en Bachillerato de CC.SS. y Humanidades). Las tareas desbordan a un solo profesional, casi siempre formado más en un campo que en otro (psicológico más que pedagógico o viceversa), y carente sin embargo de formación específica para determinadas tareas (Royo, 2002).

Quizá las funciones más desarrolladas en los últimos años han sido la coordinación y el asesoramiento. Un ejemplo de ello es la labor realizada en el propio departamento, en especial las: reuniones generales de equipo y las reuniones específicas con profeses de diversificación, aulas especiales, etc., todos ellos pertenecientes al D.O.

El asesoramiento ha avanzado de tal manera que se han generalizado las reuniones del orientador con el equipo directivo, los coordinadores de ciclos, los departamentos y los seminarios. Por otra parte, las reuniones con profesionales ajenos al sistema educativo se están ampliando para abarcar a las Departamentos de Bienestar Social, a los educadores de barrio y de minorías étnicas, responsables de centros de procedencia de los alumnos, y responsables de programas de salud y prevención de instituciones supramunicipales. Los orientadores tienen, en consecuencia, demasiadas reuniones como para poder prepararlas debidamente, así como para poner en práctica las conclusiones que de ella se derivan.

Pero el trabajo con el alumnado sigue siendo central, como lo fue desde el principio. El profesorado y los tutores piden intervenciones directas con las clases y los alumnos con dificultades. Sin embargo, el aumento de la diversidad del alumnado y "sus derechos" propician un notable aumento de la demanda en asesoramiento y orientación, de tal manera que el alumnado que parecía no tener "problemas" ni "necesidades" especiales, comienza a solicitar ayuda directa y cercana, tanto sobre la orientación profesional como sobre la situa- 
ción personal. Sólo esta demanda sería suficiente para justificar la presencia de un orientador en muchos de los centros.

Pero además, en los institutos existen otros alumnos pertenecientes a aulas muy diferentes: de apoyo, de diversificación curricular, de grupos especiales..., y son justamente estas aulas las que reclaman mayor atención. Todos estos alumnos deben ser sistemáticamente evaluados como condición previa para acceder a dichas aulas.

Por otra parte, la petición de asesoramiento de las familias es proporcional a la demanda del alumnado, si tenemos en cuenta que en muchos casos la necesidad de asesoramiento a las familias proviene de la necesidad de orientación manifestada por el alumno. En otras ocasiones, es la propia familia la que desea recibir información fiable sobre los problemas (y las posibles soluciones) que surgen en el propio contexto familiar.

Uno de los elementos distintivos de los D.O. en España es su fuerte apoyo a las tutorías en los centros escolares, debido a la idea de que el profesorado puede orientar a los alumnos y sus familias. Y esta idea, peculiar de nuestro sistema educativo, nos diferencia de otros países europeos. Se trata, con todo, de una verdad a medias, ya que en la mayor parte de los casos son también docentes, y como parte implicada no siempre resultan tan objetivos como sería deseables.

Los tutores pueden intentar realizar dichas funciones siempre que cuenten con la debida preparación, y así suele ser normalmente. Pero la función de orientación requiere una formación de base específica; más aún: los cambios tecnológicos y la propia globalización acabarán forzando dicha especialización.

Aunque los docentes no siempre lo vean, los orientadores siguen empleando una buena parte de su tiempo en la formación del profesorado en lo que a la función tutorial se refiere. Otros países, por el contrario, han optado por especialistas para dichas tareas, dejando a los tutores las funciones de integración de los grupos con los que trabajan.

Es obvio que tal cantidad de funciones para un solo profesional no pueden ser bien atendidas. Hasta ahora se había creído que abarcar muchos campos y tareas podía llevar a afianzar más el perfil orientador. Pero la realidad se impone: la sociedad demanda tareas específicas y especializadas, y esto implica distribuir las funciones entre distintos perfiles profesionales. Corremos así el riesgo de querer abarcar mucho, pero profundizando poco, una actitud a la larga contraproducente. Y es entonces cuando "el mago se queda sin magia" (Selvini, 1996) y los alumnos, padres y profesores sin la orientación que necesitan.

\section{Tendencias de futuro en los D.O}

\section{Mayor cantidad de reuniones de coordinación}

Si en la actualidad el número de reuniones es ya considerable, en el futuro aumentará. La sociedad y el sistema educativo piden cada vez más consejo a los expertos, y las decisiones se tomarán en equipo. Las coordinaciones se harán con los miembros del propio departamento, con los equipos de aulas específicas (diversificación, apoyo...), con los equipos directivos, con los departamentos de calidad, con los responsables de ciclos y etapas, con el profesorado específico de Formación y Orientación Laboral, con los equipos municipales de trabajo social, los responsables de organismos y entidades municipales y supramunicipa- 
les, los responsables de centros de procedencia de nuevo alumnado..., y por supuesto con los centros de asesoramiento externo (Moya, 2001).

\section{Mayor demanda de asesoramiento personalizado en orientación profesional y "para la carrera" para el alumnado y sus familias}

El alumnado y sus familias exigirán orientación e información sobre itinerarios, asignaturas y estudios, tanto para dentro del Estado como fuera de él. Esta área se especializará cada vez más y será demandada a corto y largo plazo por todos los grupos. Los institutos que pretendan ofrecer calidad deberán cuidar este apartado combinando las actividades grupales con las personales, pues la orientación profesional está regida por el principio de ser "personal y única" para cada sujeto.

\section{Asesoramiento y orientación con las nuevas tecnologías de la información y comunicación}

La información actualizada será la base de una buena orientación. Los alumnos y sus familias pedirán asesoramiento a través de canales informáticos (correo electrónico, páginas web, chat...) (Benavent, 1999, Pantoja, 2002, Villa, 2002). Por otra parte, la información y los materiales de apoyo llegarán al profesorado a través de sus buzones de correo electrónico.

\section{Mayor demanda del alumnado y sus familias de asesoramiento personal $y$ terapia breve en los centros docentes}

Los alumnos tienen cada vez más cerca a sus orientadores, y buscarán soluciones a sus necesidades y problemas en los D.O. antes que en cualquier otro centro de salud especializado. Los centros deberán pensar en ofrecer estos servicios para aportar calidad a sus alumnos

\section{Mayor demanda de orientación y asesoramiento a profesores y tutores}

La diversidad de los alumnos forzará a los profesores a buscar nuevas y más eficaces estrategias de actuación. No podrán ser ajenos a la demanda de asesoramiento para poder centrar sus actuaciones y en especial para poder afrontar sus dificultades.

Aumentará el movimiento de profesores debido a las necesidades de actualización didáctica (informática, segundos y terceros idiomas, didácticas específicas, tecnologías de la información...), e igualmente se diversificará. Habrá más profesorado joven (inexperto pero bien preparado) que cubrirá la formación de otros; profesorado experto y profesorado de más edad (con más rigidez a los cambios). Todo esto llevará a los orientadores a ocuparse del profesorado con la intención de adaptar al nuevo ambiente a los inexpertos, apoyar a los más rígidos y a soportar el "síndrome de quemado" de todos ellos. Los centros y la administración educativa optarán por apoyar a su profesorado, evitando mayor número de bajas laborales y favoreciendo así una política preventiva de riesgos laborales. 


\section{Demanda administrativa y social de los procedimientos de calidad... también a los departamentos de orientación}

Los procedimientos de calidad se imponen en los sistemas europeos, y aunque la institución escolar sea crítica con ellos, tendrá que terminar por incorporarlos. Esos procedimientos afectarán a los D.O. y obligarán a aumentar los tiempos de planificación y evaluación de las tareas desempeñadas. Y sobre todo los clientes del D.O. (alumnado, familias y el propio profesorado), forzarán a trabajar con mayor calidad, orden y planificación. Será cada vez más difícil "querer hacerlo todo", ya que la calidad obligará a realizar con más profundidad tareas que ahora realizamos de forma más rápida y sin planificar.

\section{Mayor creatividad y flexibilidad en la organización de aulas, grupos de alumnos, itinerarios, ...}

Si se quiere llegar con calidad a la diversidad del alumnado, las soluciones generales no servirán para poder atender a colectivos tan diferentes (emigrantes, minorías étnicas, grupos con baja motivación y distintas capacidades...). Los centros y los D.O. se verán involucrados en la creación de nuevas estrategias que se adapten a la diversidad. El D.O. será agente activo en dicho proceso.

\section{Mayor nivel de iniciativas y propuestas para la prevención de problemas y necesidades que van a ir surgiendo}

En este momento la "convivencia" en los centros se muestra como uno de los puntos más importantes, pero surgirán otros nuevos como la integración y la convivencia con las minorías, la desmotivación por los estudios de rango superior que requieren mayor esfuerzo, la diferencia entre alumnado con o sin acceso a las nuevas tecnologías de la información y la comunicación, y otros temas que los alumnos vivirán en sus familias y que repercutirán directamente en la educación, como la afectividad y la sexualidad, la diferencia en la cantidad de conocimiento e información...

\section{Mayor demanda de asesoramiento de los órganos que dirigen los centros educativos}

Los equipos directivos pedirán asesoramiento sobre organización escolar, estructura de grupos de clase, implementación de las nuevas tecnologías..., y utilizarán para ello a los D.O. Ello llevará a la existencia de más perfiles profesionales con formación básica superior en los D.O., que se coordinarán con órganos de dirección, jefaturas de estudio, responsables de calidad y responsables de departamentos didácticos.

\section{Mayor coordinación con los agentes externos al sistema educativo}

La diversidad de los alumnos, muchos de ellos conectados a las redes de atención social y comunitaria de las instituciones locales, llevarán a planificar y a contactar con educadores y trabajadores sociales, que hacen funciones de intermediación entre los centros, la calle y las familias. 


\section{Perfiles y organización de los departamentos de orientación. Un planteamiento para los próximos años}

\section{La configuración actual de los Departamentos de Orientación en España. Una apuesta positiva hacia el futuro}

Los D.O. en España han tenido una peculiaridad muy importante: incorporar prácticamente en una sola figura las tareas de orientación, asesoramiento, tratamiento, formación y evaluación. Tanto al alumnado con n.e.e. propiamente, como sin ellas. Y estas tareas han recaído sobre los orientadores de los centros de secundaria. El resto de las funciones especialmente docentes han recaído sobre diferentes perfiles que a continuación se detallarán.

Por tanto, los D.O. están formados por aproximadamente 6/7 miembros en centros de unos 700 personas. Estos departamentos suelen tener los siguientes componentes de base:

a) 1 profesor orientador/a., responsable del D.O., con requisito de psicología, pedagogía, o psicopedagogía

b) $1 / 2$ profesores/as de apoyo a las n.e.e., con requisito de magisterio educación especial, y/o magisterio audición y lenguaje.

Además en algunos centros con otras necesidades existen otras figuras:

c) 1 trabajador social (sólo en algunas Comunidades Autónomas)

d) 2 profesores de aula de Diversificación Curricular, para cursos específicos con alumnado de 16-18 años, con requisito de licenciatura

e) $1 / 2$ profesores para apoyo a otras aulas específicas (sólo cuando hay cursos específicos con problemas especiales de aprendizaje/adaptación, con requisito de diplomatura y/o licenciatura

f) 1 profesor de Formación y Orientación Laboral (cuando hay ciclos formativos profesionales, con requisito de licenciatura en diferentes áreas).

Apoyamos desde este artículo el mantenimiento dentro de los D.O. de estos perfiles y otros nuevos que van a ir apareciendo (educadores/as sociales, ...).

\section{Nuevos perfiles para la orientación, coordinación, formación y evaluación psicopedagógica. La nueva "apuesta" para un correcto funcionamiento de los D.O. en el futuro}

Actualmente de todas los perfiles enumerados, solamente uno es responsable de la coordinación, asesoramiento, orientación y evaluación psicopedagógica: el profesor orientador. $\mathrm{El}$ resto de las personas tienen tareas docentes. Las tareas docentes quedan cubiertas, pero no así las tareas orientadoras. Además ninguna de la titulaciones (por sí solas) que dan la formación base a la figura del orientador/a cubren de lleno las necesidades actuales y las futuras. Proponemos varios perfiles:

\section{Perfil de Psicopedagogía}

Entendiendo que el psicopedagogo es el profesional capacitado para la orientación e intervención educativas, destinado a promover el desarrollo integral de la persona, en su rea- 
lidad individual y social, y el desarrollo óptimo de los procesos de aprendizaje (individuales y grupales) en el marco de la mejora de la calidad educativa (Fernández A. y otros 2002) este tendría entre sus principales funciones en los centros de Educación Secundaria:

a) asesoramiento, orientación y formación a tutores en sus tareas con el profesorado de su grupo, el alumnado y sus familias

b) asesoramiento, orientación y formación al resto del profesorado en el campo de las necesidades educativas especiales (n.e.e.). y la diversidad cultural y social del alumnado

c) coordinación del profesorado que trabaja en aulas diferenciales como: aulas de diversificación curricular, aulas de tareas, aulas de apoyo y otras aulas específicas

d) evaluación del alumnado en competencias escolares

e) orientación a las familias en temas relacionados con el aprendizaje de sus hijos/as.

\section{Perfil de Psicología}

Extrapolando algunas de las funciones que se manifiestan en los perfiles profesionales del Colegio Oficial de Psicólogos para el ámbito educativo (COP 1998), entre sus principales funciones dentro de los centros de Educación Secundaria estarían las siguientes:

a) evaluación del alumnado en áreas personales, cognitivas, familiares y sociales

b) asesoramiento, consejo y ayuda psicológica breve al alumnado en general

c) asesoramiento breve a las familias en temas psicológicos (dificultades personales, familiares) y en estrategias de ayuda psicológica a sus hijos/as

d) asesoramiento, consejo y ayuda psicológica breve al profesorado, en temas relacionados con su motivación laboral y situaciones personales, que afecten directamente a su docencia

e) docencia de la asignatura de psicología en el bachillerato.

\section{Perfil de Psicología y/o Psicopedagogía indistintamente}

Algunas de las funciones corresponderían al perfil de psicología y psicopedagogía indiferentemente:

a) evaluación grupal e individual del alumnado del centro (psicológica y pedagógica)

b) orientación profesional y para la carrera de los alumnos/as

c) seguimiento psicológico y pedagógico del tránsito del alumnado entre etapas y diferentes centros educativos.

\section{Perfil de Pedagogía}

La pedagogía ayuda al análisis, organización y desarrollo de sistemas/procesos educativos. En general los pedagogos se ocupan de las técnicas y métodos de enseñanza, de su evolución, y de la investigación de nuevos procesos didácticos. Realizan trabajos de formación del profesorado y supervisan los servicios de carácter educativo (Auzmendi E., Beza- 
nilla M. J., Elespuru I., Pereda V. 2002; Casares 2000). Entre sus principales funciones en el centros de Educación Secundaria estarían las siguientes:

a) orientación y consejo al equipo directivo en temas de organización escolar

b) orientación didáctica al profesorado

c) desarrollo e implementación de Programas Educativos dentro del contexto escolar

d) asesoramiento en implementación de Programas Específicos relacionados con las nuevas Tecnologías de la Información y la Comunicación al centro y a todo el profesorado

e) coordinación pedagógica de los ciclos educativos de secundaria y la relación de la etapa secundaria obligatoria con la post-obligatoria

f) planificación de programas específicos de formación para las familias

\section{Perfil de Trabajo Social}

Entre sus principales funciones en el centros de Educación Secundaria estarían las siguientes:

a) seguimiento de alumnado (familia, centro, instituciones externas) con problemática social

b) búsqueda de recursos, asociaciones y colectivos de inserción para el alumnado, que favorezcan su desarrollo e integración social en horario extraescolar

c) coordinación de actividades para el alumnado con inadaptación social en horarios no docentes, dentro de los centros educativos

d) búsqueda de recursos económicos y financieros de instituciones para la ayuda a la escolarización del alumnado con problemática social

e) evaluación del alumnado con necesidades familiares y sociales, cuya integración escolar esté afectada

f) seguimiento del alumnado en "programas a medio camino" entre el centro escolar y otras instituciones educativas (garantía social, ...).

\section{Algunas preguntas y respuestas a las funciones orientadoras}

Acabamos de indicar los principales perfiles profesionales que deberían incorporarse a los D.O. Hemos visto que son profesionales específicamente preparados para tareas de coordinación y asesoramiento, que actuarán sobre tareas que actualmente están cubiertas de manera deficitaria e incompleta.

Algunas indicaciones se deben hacer ahora que se han indicado estos perfiles:

\section{¿Cuántas figuras deben estar en las funciones técnicas orientadoras?}

En los centros con menos de 200 alumnos será suficiente una figura orientadora. Aquellos que tengan entre 200-500 alumnos deberían tener dos. En los centros con gran número 
de alumnado debieran coexistir todas ellas (psicólogo, psicopedagogo, pedagogo, y trabajador social) ya que la población sobre la que intervienen será lo suficientemente diversa.

\section{¿Qué figura es más importante si tenemos que decidir entre unas y otras?}

Los centros deben valorar en función de sus necesidades las figuras que prioritariamente deben existir. Lo lógico es que coexistan al menos psicopedago y psicólogo. Las características del alumnado, junto con los objetivos del centro, son los elementos que deben valorarse para tomar esa decisión. Lo importante es la coexistencia de diferentes perfiles en diferentes tareas, evitándose dos perfiles con la misma formación académica.

\section{¿Cada figura trabaja sólo en sus tareas?}

El profesorado, alumnado y familias deben tener claro las funciones que desempeñan cada figura orientadora. Los clientes reciben mal la mezcla de las funciones sobre temas didácticos, psicopedagógicos, y psicológicos. Sin embargo, las diferentes figuras se coordinarán para plantear actuaciones generales sobre los centros y evaluar posteriormente sus actuaciones; dándose ayuda y soporte cuando sea necesario.

El organizador básico que configura los D.O. es el grupo de trabajo que interacciona y construye la tareas que van a realizar: el resultado no es la suma de lo que aporta cada uno de sus miembros, sino la propia dinámica que se establece entre los participantes y la tareas.

\section{¿Es un modelo de intervención clínico, psicopedagógico, centrado en programas, ...?}

Es un modelo de intervención centrado en las tareas, donde se combinan de manera organizada la clínica, la consulta, el desarrollo de programas, el consejo, y el asesoramiento en temas didácticos y organizativos. Es un modelo mixto, donde el componente tecnológico y humanista interaccionaran de manera dinámica. Y donde las diferentes disciplinas relacionadas con la educación aportan lo mejor de sus saberes.

\section{¿Qué figura será la responsable de dirigir los Departamentos de Orientación?}

Cualquiera de las figuras descritas puede ser la persona responsable del D.O., siempre que su formación y experiencia haya sido en áreas psicoeducativas.

\section{¿Qué relación mantendrán esas figuras con el resto de los componentes del Departamento de Orientación que se dedican fundamentalmente a tareas docentes?}

Los profesionales con funciones orientadoras darán consejo, asesoramiento y elaborarán propuestas con el resto de los componentes del D.O. dedicados a tareas docentes. Según las demandas, se reunirán con cada componente del D.O. de manera particular. En ocasiones excepcionales se articularán reuniones comunes para realización y coordinación de proyectos específicos. 


\section{Conclusiones}

En este artículo se plantea la necesidad de "reorientar" el trabajo desarrollado por las figuras orientadoras. Hasta ahora, estas figuras provenían de diferentes campos del saber (psicología, psicopedagogía, pedagogía). Y esto es positivo.

Pero todas ellas han tenido asignadas las mismas funciones para su cargo: funciones diseñadas originalmente por las Administraciones educativas en los años ochenta. Además, estas funciones han sido excesivas y se han visto desbordadas por los cambios producidos en los últimos años: la globalización, la interculturalidad ... Esto ha hecho que la población a la que atienden (profesorado, alumnado y familias) dejen de creer en su capacidad de asesoramiento: los psicólogos desconocen los aspectos pedagógicos, los psicopedagogos el asesoramiento psicológico y los pedagogos están principalmente formados en áreas de organización escolar, didáctica y educación especial.

Por eso, en este artículo se plantea que los D.O. tengan varias figuras, cada una de ellas formada en un campo del saber, que se centre en aquello para lo que se formó, y realice sus tareas con especialización. Eso ayudaría a las propias figuras y a sus clientes a centrarse en sus funciones, y evitaría la dispersión de tareas que existen en la actualidad. Por ello, la contratación debiera basarse en los diferentes perfiles (psicología, pedagogía, psicopedagogía y trabajadores sociales), que se irían incorporando a los D.O.

A pesar de la especialización, los diferente perfiles deberán coordinarse, interaccionar y plantear estrategias en común. La aportación de cada uno de ellos enriquecerá las actuaciones que se realicen desde el D.O.

\section{Bibliografía}

Alvarez, M. (1999). Diversidad y confluencia. Un estudio interpretativo de los centros educativos en la Unión Europea. Bilbao: INCE e ICE de la Universidad de Deusto.

Alvarez, M. (2002). "Formación y desarrollo profesional en el marco europeo". En Maiztegui, C. y Santibáñez, R. (2002). El futuro del educador. Perfiles profesionales y adaptación de los pedagogos y educadores sociales a una sociedad en cambio. Bilbao: Universidad de Deusto.

Auzmendi, E., Bezanilla, M. J., Elespuru, I. y Pereda V. (2002). "El perfil del pedagogo". En Maiztegui, C. y Santibáñez, R. (2002). El futuro del educador. Perfiles profesionales y adaptación de los pedagogos y educadores sociales a una sociedad en cambio. Bilbao: Universidad de Deusto.

Benavent, J. A. (1999). "La orientación psicopedagógica en el umbral del siglo XXI". Revista de orientación y psicopedagogía, Vol. 10, n 17, 53-62.

Bisquerra, R. (2002). "Pasado, presente y futuro de la psicopedagogía". En Martinez de la Hidalga Z. (coord.). Definición y perspectivas profesionales de la psicopedagogía. Bilbao: Universidad de Deusto.

Boza, A. (2001). "Los equipos de orientación de sector. Funciones y modelos de intervención". Revista española de orientación y psicopedagogía. Vol., 12, n 21, $1^{\circ}$ semestre, pp. 50-71.

Casares, P. (2000). "Las salidas profesionales como criterio de calidad en la licenciatura de Pedagogía”. Bordon, 52 (4), pp. 499-508.

Colegio Oficial de Psicólogos (1998). Perfiles profesionales. Psicología de la educación. http://www.cop.es/perfiles/contenido/educativa.htm (30-7-03). 
Del Rincón, B. (2001). Presente y futuro del trabajo psicopedagógico. Barcelona: Ariel.

Fernández Gonzalez, A., López Paz, J. F., Martínez de la Hidalga, Z., Villardón Gallego, L. y Yaniz Alvarez de Eulate, C. (2002). "El perfil profesional de la psicopedagogía e implicaciones para el futuro". En Martínez de la Hidalga, Z. (coord.). Definición y perspectivas profesionales de la psicopedagogía. Bilbao: Universidad de Deusto.

Iturbe, T. y Del Carmen, I. (1973). El departamento de orientación en un centro escolar. Madrid: Narcea.

Maiztegui, C. y Santibáñez R. (2002). El futuro del educador. Perfiles profesionales y adaptación de los pedagogos y educadores sociales a una sociedad en cambio. Bilbao: Universidad de Deusto.

Martínez de la Hidalga, Z. (coord.) (2002). Definición y perspectivas profesionales de la psicopedagogía. Bilbao: Universidad de Deusto.

MEC (1989). Libro Blanco para la reforma del sistema educativo. Madrid: MEC.

MEC (1990). La orientación educativa y la intervención psicopedagógica. Madrid: MEC.

MEC (1992). Orientación y tutoría. Madrid: MEC.

Motoso, J. (2002). "Innovación psicopedagógica en un contexto escolar público de educación secundaria”. En Martínez de la Hidalga, Z. (coord.). Definición y perspectivas profesionales de la psicopedagogía. Bilbao: Universidad de Deusto.

Moya, A. (2001). "El profesor de apoyo a la integración: un perfil a redefinir mediante la colaboración”. Revista española de orientación y psicopedagogía, Vol. 12, n² 21, 1 semestre, pp. 109-118.

Pantoja, A. (2002). "El modelo tecnológico de intervención psicopedagógica". Revista española de orientación y psicopedagogía, Vol. 13, n 2, $1^{\circ}$ semestre, pp. 189-210.

Royo, F. (2002). "Desempeño profesional de los orientadores en los institutos de educación secundaria de Salamanca. Creación de un instrumento de valoración y autovaloración". Revista Española de Orientación y Psicopedagogía, Vol. 13, nº 1, pp. 31-50.

Sanz Oro, R. (2001). Orientación psicopedagógica y calidad educativa. Madrid: Pirámide.

Selvini, M. (1996). El mago sin magia: como cambiar la situación paradójica del psicólogo en la escuela. Barcelona: Paidos.

Sobrado, L. (1998). "Servicios externos de orientación a los centros educativos: los modelos de las comunidades autónomas". Revista española de orientación y psicopedagogía, Vol. $9, \mathrm{n}^{\circ} 15,1^{\circ} \mathrm{se}-$ mestre, pp. 109-132.

Solé, I. (1998). Orientación educativa e intervención psicopedagógica. Barcelona.

Solé, I. (1999). "Intervención psicopedagógica: una o — ¿más de una? — realidad compleja”. Infancia $y$ aprendizaje, 87, 9-26 ICE.UB. Horsori.

Villa, A. (2002). "Los retos actuales de la educación y su repercusión en la formación del educador". En Maiztegui, C. y Santibáñez R. (2002). El futuro del educador. Perfiles profesionales y adaptación de los pedagogos y educadores sociales a una sociedad en cambio. Bilbao: Universidad de Deusto. 